\title{
Coordenação do cuidado e ordenação nas redes de atenção pela Atenção Primária à Saúde - uma proposta de itens para avaliação destes atributos
}

\author{
Evaluation of health care coordination and health care networks ordination by Primary Health \\ Care - a proposal of items for the evaluation of these attributes
}

\section{Coordinación del cuidado y clasificación en las redes de atención por la Atención Primaria de Salud - una propuesta de ítems para la evaluación de estos atributos}

Patricia Sampaio Chueiria ${ }^{2}$ Erno Harzheimª, Silvia Maristela Pasa Takeda ${ }^{b}$

\section{Resumo}

Introdução: A formação de redes de atenção à saúde (RAS) tem se mostrado como uma forma de organização de sistemas de saúde eficaz para responder a alguns dos atuais desafios epidemiológicos e estruturais destes sistemas. O Brasil vem caminhando para a regionalização e a conformação de redes de atenção à saúde. A fim de avaliar o desenvolvimento das RAS, propõe-se um conjunto de itens para inferir dois dos atributos das redes: a coordenação do cuidado individual e a ordenação das RAS pela Atenção Primária à Saúde. Métodos: Utilizou-se o Método Delphi, composto pelas seguintes etapas: definição inicial dos itens a serem avaliados e composição do grupo de experts, realização de quatro rodadas do Delphi e avaliação dos resultados. Resultados: O produto final foi uma lista de 69 itens, destes, 59 questões atingiram estabilidade de opinião entre os experts e podem ser divididas em: um grupo de 44 itens que atingiram grau de consenso alto entre os experts para inclusão do item na lista final; um grupo de 12 itens que atingiram grau de consenso intermediário; e um grupo de 3 itens que atingiram grau de consenso baixo. A construção desta proposta de itens para avaliação das RAS tem por intuito central colaborar com as pesquisas relacionadas às avaliações sistêmicas dos serviços de saúde.

\begin{abstract}
Introduction: The creation of health care networks $(\mathrm{HCN})$ in national health care systems has proved to be an effective form of organization of health care systems to address some of these epidemiological and structural challenges. Brazil has been moving towards the regionalization and conformation of $\mathrm{HCN}$. In order to assess the development of $\mathrm{HCNs}$, this research offers to define initial items that in the future will compose an evaluation tool capable of measuring two attributes of health care networks: individual care coordination and planning of $\mathrm{HCNs}$ by the Primary Health Care. Methods: We used the Delphi method, which involves the following steps: initial setting of items to be evaluated and composition of the experts group, conducting four rounds of Delphi and evaluation of results. Results: The final product was a list of 69 items, of these, 59 items reached stability between experts and can be divided into: a group of 44 items that achieved a high degree of consensus among the experts to include the item in the final list; a group of 12 items that reached an intermediate degree of consensus, and a group of 3 items that reached a low degree of consensus. The construction of this initial proposal of items for evaluation of $\mathrm{HCN}$ has the main goal of collaborating with researches related to the more systemic evaluations of health services.
\end{abstract}

Como citar: Chueiri PS, Harzheim E, Takeda SMP. Coordenação do cuidado e ordenação nas redes de atenção pela Atenção Primária à Saúde - uma proposta de itens para avaliação destes atributos. Rev Bras Med Fam Comunidade. 2017;12(39):1-18. http://dx.doi.org/10.5712/rbmfc12(39)1363
Palavras-chave:

Atenção Primária à Saúde

Avaliação de Serviços

de Saúde

Avaliação em Saúde

Keywords:

Primary Health Care

Health Services Evaluation

Health Evaluation

Fonte de financiamento: declaram não haver. Parecer CEP: 92.979 (UFRGS), aprovado em 10/09/2012.

Conflito de interesses: declaram não haver.

Procedência e revisão por pares: revisado por pares. Recebido em: 03/08/2016. Aprovado em: 04/06/2017. 


\section{Resumen}

Introducción: La formación de redes de atención de salud (RAS) ha demostrado ser una forma de organización de los sistemas de salud eficaz para responder a algunos de los actuales retos epidemiológicos y estructurales de estos sistemas. Brasil se ha estado moviendo hacia la regionalización y la conformación de redes de atención de salud. Con el fin de evaluar el desarrollo de las RAS, se propone elementos de ajuste para componer una herramienta que es capaz de evaluar dos de los atributos de las redes: la coordinación de la atención individual y la ordenación de RAS de Atención Primaria Salud. Métodos: Se utilizó el método Delphi, que comprende las siguientes etapas: definición inicial de los elementos a evaluar y la composición del grupo de expertos, la realización de cuatro rondas de Delphi y evaluación de los resultados. Resultados: El producto final fue una lista de 69 tópicos, de estos, 59 temas llegaron a vista de la estabilidad entre el experto y se pueden dividir en: un grupo de 44 elementos que han alcanzado alto grado de consenso entre los expertos para incluir el tema en la lista final; un grupo de 12 elementos alcanzó grado intermedio de consenso; y un grupo de tres artículos que alcanzaron bajo grado de consenso. La construcción de esta propuesta inicial de elementos para la evaluación de RAS tiene el objetivo central de colaborar con las investigaciones relacionadas con evaluaciones sistemáticas de los servicios de salud.
Palabras clave:

Atención Primaria de Salud

Evaluación de

Servicios de Salud

Evaluación en Salud

\section{Introdução}

A formação de redes de atenção à saúde tem se mostrado uma forma efetiva de enfrentamento de desafios organizacionais e assistenciais dos sistemas de saúde. ${ }^{1-4}$ Entre estes desafios, destacam-se a dificuldade de acesso, o aumento da carga de doenças crônicas e a fragmentação da oferta de ações e serviços de saúde. ${ }^{5}$ No Brasil, soma-se à oferta indiscriminada de ações e serviços o desequilíbrio de recursos humanos e financeiros entre a Atenção Primária à Saúde (APS) e a atenção especializada (ambulatorial e hospitalar), o financiamento insuficiente, as iniquidades regionais e a formação profissional inapropriada. $^{6}$

Acompanhando um processo mundial, o Brasil ruma para a criação e o fortalecimento de redes de atenção à saúde (RAS). ${ }^{4}$ Em um primeiro momento, pautado pelas diretrizes constitucionais de descentralização e regionalização, e, a seguir, avançando em um processo normativo apoiado na conformação de regiões de saúde e na definição de responsabilidades de cada esfera de gestão., ${ }^{3,7-13}$ Observa-se, porém, incipiente progresso na integração entre o planejamento e a organização dos serviços de saúde e a execução das ações. ${ }^{3,7-13}$

Além da regionalização, as redes de atenção à saúde requerem uma atenção primária forte e estruturada, ou seja, que exerça os papeis de ordenação e de porta de entrada prioritária nas RAS, e responsável pela coordenação do cuidado das pessoas. ${ }^{4}$

AAPS teve uma expansão exponencial no Brasil com a Estratégia Saúde da Família nas décadas de 90 e $2000^{14-16}$ e desde 2013 também viu o interesse de diferentes atores (população, mídia e associações médicas) aumentar com a criação do Programa Mais Médicos. Contudo, ainda enfrenta a necessidade de legitimação perante à sociedade e aos gestores, necessitando melhorar o acesso, superar déficits de infraestrutura, de qualificação profissional e de resolutividade. ${ }^{17,18}$

Aimportante tarefa da APS em assumir a responsabilidade pela ordenação da rede e pela coordenação do cuidado é justificada especialmente por dois fatos. Um deles é o princípio organizativo das RAS, que deve ser constituída a partir das necessidades de saúde da população. É na APS que as pessoas são cadastradas, acompanhadas, estratificadas em relação a riscos e vulnerabilidades e têm, portanto, suas necessidades identificadas de forma mais abrangente..$^{4,19}$ 
O outro fato é a hegemonia das doenças crônicas no perfil epidemiológico e o cuidado diferenciado que estas doenças demandam. ${ }^{20}$ São situações frequentemente complexas, envolvendo diversos serviços de saúde, o que amplia a possibilidade de nova solicitação de exames, o risco de interações medicamentosas, a falta de comunicação entre os profissionais, a não corresponsabilização e subsidiariedade sobre o cuidado entre os diversos serviços, aumentando a chance de erros e complicações, e elevando o custo do cuidado desses pacientes para o sistema de saúde, para o próprio paciente, sua família e a sociedade. ${ }^{21-23}$ A coordenação do cuidado é vista, na literatura, como uma ação essencial para que os profissionais de saúde enfrentem os riscos apresentados acima. ${ }^{23}$

A organização das redes de atenção à saúde vem se dando de forma heterogênea em todo o mundo, e pode-se dizer que parte das dificuldades de intercâmbio, análise e comparação dessas estratégias deve-se à nomenclatura diversa e à amplitude de ações a que se referem. ${ }^{2,4,24} \mathrm{~A}$ fim de delimitar esse campo conceitual, diversos autores e organismos internacionais têm proposto definições e características que demarquem o conceito de redes de atenção à saúde. 1,3,25,26 $^{2}$

A Organização Mundial da Saúde (OMS) define como serviços integrados ou redes de atenção à saúde: "gestão e prestação de serviços de saúde para que os usuários recebam um contínuo de serviços preventivos e curativos, de acordo com as suas necessidades ao longo do tempo e em diferentes níveis do sistema de saúde". ${ }^{25}$ Neste sentido, em 2010, a Organização Pan-Americana da Saúde propôs 14 atributos, divididos em 4 eixos, para a caracterização das redes de atenção à saúde (Quadro 1). ${ }^{4}$

Quadro 1. Atributos das Redes de Atenção à Saúde.

\section{Eixo Modelo Assistencial}

Atributo 1 - Território e população definidos, com amplo conhecimento das necessidades e preferências em relação à saúde.

Atributo 2 - Rede de serviços extensa, que presta cuidado integral.

Atributo 3 - Atenção primária que cobre toda a população; está organizada em equipes multiprofissionais; que é a principal porta de entrada do sistema; é responsável pela coordenação do cuidado e é resolutiva, sendo capaz de resolver os principais problemas de saúde de sua população.

Atributo 4 - Prestação de serviços especializados em lugares apropriados e preferencialmente em ambientes extra-hospitalares.

Atributo 5 - Existência de mecanismos de coordenação assistencial em todos os serviços.

Atributo 6 - Atenção à saúde centrada na pessoa, na família e na comunidade, levando em conta as particularidades culturais.

Eixo Governança e estratégia

Atributo 7 - Sistema de governança único para todos os serviços.

Atributo 8 - Participação social ampla.

Atributo 9 - Ação Intersetorial e abordagem dos determinantes sociais da saúde e da equidade em saúde.

Eixo Organização e gestão

Atributo 10 - Gestão integrada de apoio clínico, administrativo e logístico.

Atributo 11 - Recursos humanos suficientes, competentes, comprometidos e valorizados.

Atributo 12 - Sistema de informação integrado que vincula todos os serviços da rede e que gera relatórios de acordo com as necessidades locais.

Atributo 13 - Gestão baseada em resultados.

Eixo Alocação de recursos financeiros e incentivos

Atributo 14 - Financiamento adequado e incentivos alinhados com as metas da rede.

Fonte: Adaptado de OPAS. ${ }^{4}$ 
A coordenação do cuidado pode ser traduzida como uma organização deliberada do cuidado individual, centrada na pessoa; com o objetivo de integrar e dar continuidade às várias ações de saúde prestadas por diferentes profissionais ou em diferentes serviços da rede. É concretizada a partir do acesso à informação, da responsabilização pelo cuidado e da organização do fluxo do usuário na rede de atenção a saúde.

A coordenação implica também em buscar garantir que o paciente receba, nos outros pontos de atenção, o cuidado que necessita, com a efetividade desejada, englobando parte dos conceitos de acesso e integralidade. Nesta pesquisa, optou-se por incluir a palavra individual após a expressão coordenação do cuidado com o objetivo de enfatizar que este conceito se refere a ações de cuidado da equipe para uma pessoa. ${ }^{19,27,28}$

Em relação à ordenação das Redes de Atenção à Saúde pela APS, pressupõe-se que o planejamento do uso dos recursos financeiros, da necessidade de formação profissional e das ações e serviços que conformam as RAS seja programado, estruturado, disposto, organizado, estabelecido, portanto, ordenado, a partir das necessidades de saúde da população. Como a responsabilidade de identificar as necessidades de saúde da população é prioritariamente da APS, assim como a demanda por outros serviços e ações em outros pontos de atenção, significa que é a partir desta ação da APS que a gestão deve ordenar as Redes de Atenção a Saúde. .,29 $^{3}$

O Quadro 2 relaciona os atributos propostos pela OPAS com conceitos e funções da APS, trazidos por Starfield e Mendes. ${ }^{3,30}$

Quadro 2. Síntese dos atributos das RAS, da APS e da função da APS nas RAS.

\begin{tabular}{|c|c|c|c|}
\hline Conceitos & Atributos das RAS (OPAS) & Atributos da APS (Starfield) & $\begin{array}{l}\text { Função da APS nas } \\
\text { RAS (Mendes) }\end{array}$ \\
\hline \multirow[t]{2}{*}{$\begin{array}{l}\text { Coordenação do cuidado } \\
\text { individual na RAS }\end{array}$} & $\begin{array}{l}\text { Primeiro nível multidisciplinar, que cobre } \\
\text { toda a população. É porta de entrada } \\
\text { ao sistema, que integra e coordena } \\
\text { a saúde, além de satisfazer a maior } \\
\text { parte das necessidades de saúde da } \\
\text { população. (atributo 3) }\end{array}$ & $\begin{array}{l}\text { Acesso } \\
\text { Primeiro contato } \\
\text { Integralidade } \\
\text { Coordenação da atenção }\end{array}$ & $\begin{array}{l}\text { Responsabilidade } \\
\text { Comunicação }\end{array}$ \\
\hline & $\begin{array}{l}\text { Existência de mecanismos de coordena- } \\
\text { ção assistencial ao longo de todo o contí- } \\
\text { nuo de serviços de saúde. (atributo 5) }\end{array}$ & $\begin{array}{l}\text { Coordenação da atenção } \\
\text { Integralidade }\end{array}$ & $\begin{array}{l}\text { Resolubilidade } \\
\text { Responsabilidade } \\
\text { Comunicação }\end{array}$ \\
\hline \multirow{2}{*}{$\begin{array}{l}\text { Ordenação da rede de } \\
\text { atenção à saúde }\end{array}$} & $\begin{array}{l}\text { População e território definido, amplo } \\
\text { conhecimento de suas necessidades } \\
\text { de saúde e preferências nas questões } \\
\text { de saúde que determinam a oferta de } \\
\text { serviços (ordenação dos serviços). } \\
\text { (atributo 1) }\end{array}$ & $\begin{array}{l}\text { Cadastro de paciente e } \\
\text { Longitudinalidade, atenção orientada à } \\
\text { pessoa fornecida ao longo do tempo } \\
\text { Acesso, Primeiro contato }\end{array}$ & Responsabilidade \\
\hline & $\begin{array}{l}\text { Sistema de informação integrado que } \\
\text { vincula todos os membros da rede, } \\
\text { com a decomposição dos dados por } \\
\text { sexo, idade, lugar de residência, etnia. } \\
\text { (atributo 12) }\end{array}$ & $\begin{array}{l}\text { Cadastro de paciente e } \\
\text { Longitudinalidade, atenção orientada à } \\
\text { pessoa fornecida ao longo do tempo } \\
\text { Coordenação da atenção } \\
\text { Acesso primeiro contato } \\
\text { Integralidade }\end{array}$ & $\begin{array}{l}\text { Responsabilidade } \\
\text { Comunicação }\end{array}$ \\
\hline
\end{tabular}

Fonte: Autoria própria, 2013.

Na revisão da literatura sobre a avaliação de RAS, não foi possível identificar um instrumento validado que visasse medir a presença e o grau de consolidação dos atributos das RAS, nem o papel da APS nas redes. 
Neste sentido, este artigo tem como objetivo propor um conjunto de elementos-chave que permitam avaliar a coordenação do cuidado individual e o papel de ordenação das redes de atenção à saúde pela APS para no futuro compor um instrumento de avaliação das redes.

\section{Métodos}

Pela revisão da literatura não sistematica, foram identificados elementos postulados como constituintes das RAS e importantes para que a APS coordene o cuidado e ordene a RAS. Estes elementos integraram a primeira versão do instrumento a ser testado, conforme descrito abaixo.

A revisão da literatura foi efetuada por meio dos seguintes termos de busca: integrated health care systems; care continuity; case management; integration of health care delivery; healthcare evaluation mechanisms; redes de atenção à saúde; avaliação de redes de atenção à saúde; coordenação do cuidado; ordenação das redes de atenção à saúde. As seguintes bases de dados foram utilizadas: PubMed e SciELO.

Realizou-se, a seguir, um estudo de busca de consenso por meio do método Delphi, que consiste na submissão iterativa de uma lista de itens temáticos a um grupo de experts, a fim de que este grupo alcance uma opinião consensual em relação a um conjunto de elementos propostos. ${ }^{31}$

O grupo de experts foi definido por indicações das seguintes instituições: o Departamento de Atenção Básica e o Departamento de Articulação de Redes de Atenção à Saúde do Ministério da Saúde Brasileiro; a Organização Pan-Americana de Saúde (OPAS); a Sociedade Brasileira de Medicina de Família e Comunidade; a Associação Brasileira de Enfermagem; o Conselho Nacional de Secretários Municipais de Saúde (CONASEMS); o Conselho Nacional de Secretários de Saúde (CONASS); e pesquisadores com experiência no tema.

Os critérios para indicação dos participantes do Método Delphi foram ser profissionais de saúde atuando na assistência (prática clínica da APS ou serviço especializado), na academia (docentes, pesquisadores, preceptores que produzam conhecimento e ensinem na área da gestão ou APS) ou em uma das três esferas de gestão do SUS envolvidas com APS ou gestão das RAS e estejam atuando no seu campo no mínimo há dois anos. Orientou-se, também, que as indicações considerassem as diversas regiões do país, com o objetivo de que o grupo fosse o mais representativo possível da realidade brasileira.

Os profissionais indicados foram convidados a participar da pesquisa Delphi por meio de correio eletrônico, e aqueles que aceitaram participar receberam cópia do projeto; o cronograma das rodadas do Delphi; e o Termo de Consentimento Livre e Esclarecido.

Foram realizadas quatro etapas do Delphi, chamadas de rodadas. Cada rodada teve a duração de 21 dias, incluindo todos os passos descritos na Figura 1. A cada rodada era enviada de forma individual e anônima para cada expert a estatística das rodadas anteriores, o que possibilitava que os participantes comparassem sua opinião com a opinião do grupo e refletissem sobre sua opinião na próxima rodada. Isso propiciava que a cada rodada o consenso em torno das questões do instrumento fosse aumentando e a estabilidade das respostas fosse elevando (Figura 1).

Durante cada rodada, o participante deveria opinar em manter ou excluir cada um dos itens. Apenas na primeira rodada permitiu-se que fossem sugeridos novos aspectos e/ou modificada a redação dos itens em análise. 


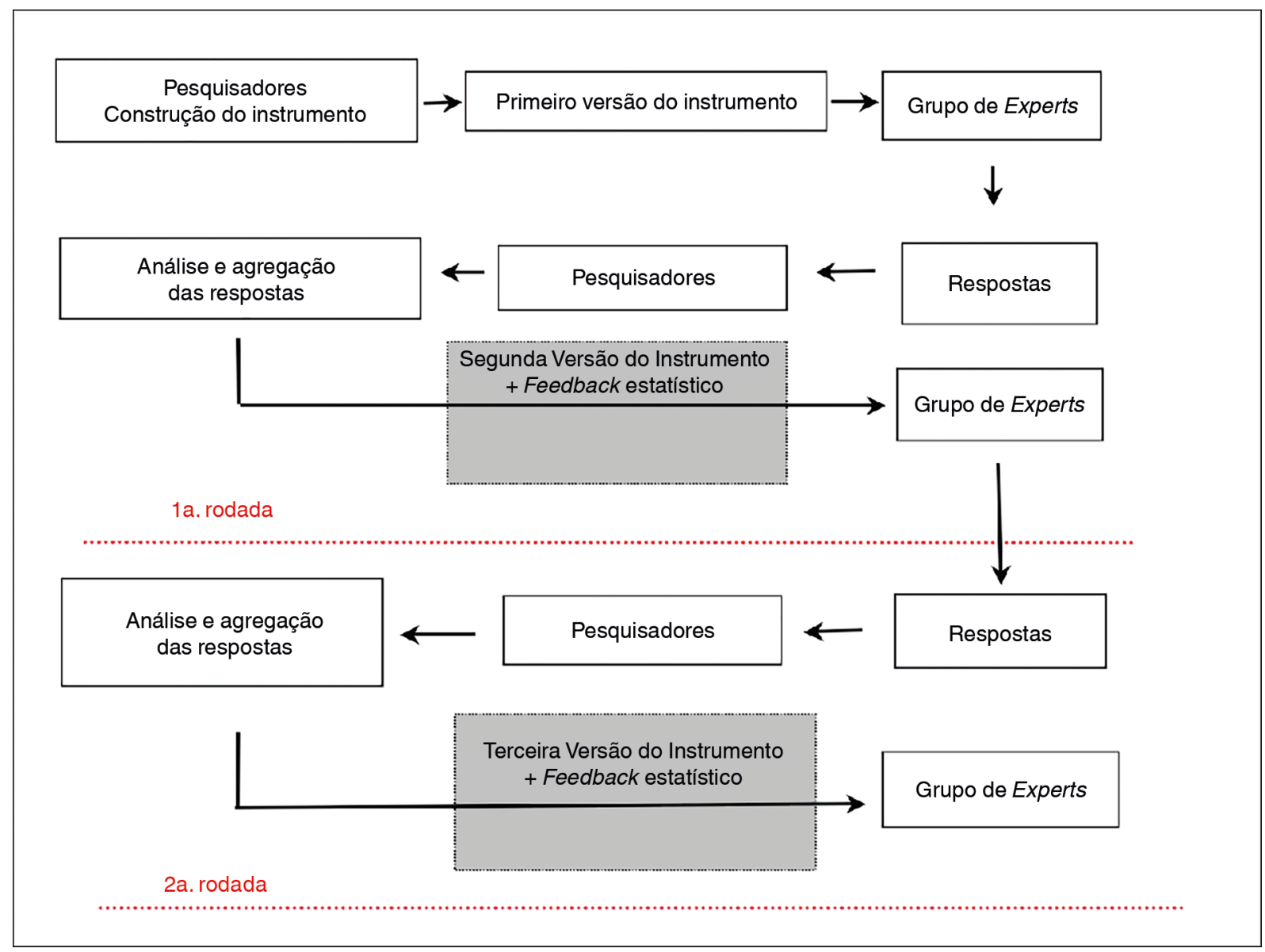

Figura 1. Fluxograma ilustrativo da metodologia Delphi. Fonte: Adaptado de Fontanive..$^{32}$

Após cada rodada, fez-se a análise estatística das respostas para cada um dos itens, utilizando-se o software Excel ${ }^{\circledR}$, e incluiu-se o percentual de participantes que optaram por manter cada item no instrumento (grau de consenso), o percentual que mudou de opinião (estabilidade das respostas) e a opinião do próprio participante na rodada anterior.

A avaliação estatística realizada entre a primeira e a segunda rodada não teve caráter eliminatório de itens, pois há necessidade de estabilização das opiniões, o que exige, no mínimo, duas rodadas de avaliação sobre os mesmos itens. A análise da primeira rodada também incluiu a decisão dos pesquisadores em relação à inclusão dos novos itens sugeridos pelos experts (esta decisão foi baseada na revisão de literatura e na experiência com o tema). Assim, adequou-se a primeira versão do instrumento de acordo com a avaliação inicial do grupo, o que levou à criação da segunda versão.

Definiu-se como estabilidade nas respostas quando a oscilação da opinião do grupo em relação a cada item fosse inferior a $10 \%$ entre duas rodadas consecutivas, e como alto grau de consenso quando o item apresentava mais de $90 \%$ de concordância em relação à inclusão do item/questão no instrumento; considerou-se grau de consenso intermediário sempre que a concordância ficasse entre 60 e $90 \%$; e baixo grau de consenso quando a concordância estivesse abaixo de $60 \%$. 
Quando o item atingia estabilidade de resposta ( $<10 \%$ de mudança de opinião), observava-se o grau de consenso; se este era de alto grau, o item era excluído das rodadas seguintes, por poder ser incorporado já naquele momento à lista de itens que foi proposta ao final do estudo. Se não houvesse alto grau de consenso, ou seja, se houvesse grau de consenso intermediário ou baixo, com estabilidade ou não na resposta, o item era mantido por mais uma rodada, e se não alcançasse consenso nesta segunda tentativa, era excluído das rodadas seguintes.

O projeto foi aprovado em 10 de setembro de 2012 pelo Comitê de Ética da Universidade Federal do Rio Grande do Sul, sob o número de Parecer 92.979.

\section{Resultados e Discussão}

Foram indicados 50 profissionais para compor o grupo de experts do Delphi; destes, 39 aceitaram participar do estudo. O perfil detalhado dos experts está descrito na Tabela 1.

O Delphi foi feito em quatro rodadas. Na primeira, 100\% (39) dos experts participantes responderam o instrumento; na segunda, 38 responderam; e, na terceira e quarta rodadas, 37 responderam.

$O$ instrumento da primeira rodada era constituído de 58 questões, divididas em 5 dimensões, similares aos principais atributos das RAS, porém relacionados aos atributos objetos da avaliação. Na segunda rodada, foram incluídas 11 questões novas e 18 (31\%) questões já existentes foram adaptadas quanto à forma, por sugestões dos participantes. Nenhuma sugestão de inclusão dos experts foi vetada. Deste modo, a segunda versão do instrumento passou a conter 69 itens. Abaixo, segue a discussão e apresentação dos resultados mais relevantes subdivididos nas cinco dimensões do instrumento. Na Tabela 2 estão descritos detalhadamente os resultados de cada item.

População e território

A primeira dimensão é composta por 10 questões. Destas, três (questões 1, 3 e 8) atingiram, já na segunda rodada, estabilidade e grau de consenso alto. O consenso relacionado à questão 3 é o que chama mais atenção, pois explicita a consolidação do papel da APS como principal porta de entrada do sistema. Duas questões atingiram estabilidade somente na quarta rodada, uma com alto grau de consenso (q5) e outra com grau de consenso intermediário (q6),e ambas tratam da adscrição populacional para a atenção especializada.

Do total de dez questões desta primeira dimensão, quatro se mantiveram instáveis até última rodada. Destas, duas, que abordavam o reconhecimento pelas equipes de saúde de suas funções e responsabilidades na RAS, chamam a atenção (q4 e q8.2), pois, apesar do consenso intermediário dos experts, vários autores reconhecem a importância das equipes conhecerem suas responsabilidades tanto para a avaliação de seu desempenho como para o desenvolvimento de sistemas de saúde. 3,4,10,22,24 Diferentemente da opinião dos experts, para os pesquisadores é manifesta a relevância destas perguntas, pois somente a partir da avaliação do trabalho desenvolvido pelas equipes de saúde e do reconhecimento das suas responsabilidades será possível avançar na melhoria do cuidado e no maior impacto nos indicadores de saúde. 
Tabela 1. Características sociodemográficas e de atuação profissional dos experts participantes do Delphi, Porto Alegre, 2013.

\begin{tabular}{|c|c|c|c|c|}
\hline Variáveis & $\begin{array}{c}\text { Profissionais } \\
\text { da gestão } \\
n=21(53.8 \%)\end{array}$ & $\begin{array}{l}\text { Profissionais da } \\
\text { assistência } \\
n=9(23.1 \%)\end{array}$ & $\begin{array}{c}\text { Profissionais da academia/ } \\
\text { universidade } \\
n=9(23.1 \%)\end{array}$ & $\begin{array}{l}\text { Total de Experts } \\
n=39(100 \%)\end{array}$ \\
\hline Características & \multicolumn{4}{|c|}{ n (\%)/média (DP) } \\
\hline Idade (anos) & $44(10,8)$ & $37,2(8,8)$ & $49,3(9,2)$ & $43,7(10,6)$ \\
\hline \multicolumn{5}{|l|}{ Sexo } \\
\hline Feminino & $10(47,6 \%)$ & 0 & $6(66,7 \%)$ & $16(41 \%)$ \\
\hline Masculino & $11(52,4 \%)$ & $9(100 \%)$ & $3(33,3 \%)$ & $23(59 \%)$ \\
\hline Tempo de formação (anos) & $19,5(11)$ & $11,7(7,7)$ & $25,1(10)$ & $18,9(10,9)$ \\
\hline \multicolumn{5}{|l|}{ Campo de atuação } \\
\hline Atenção básica & $8(38.1 \%)$ & - & - & $8(20,5 \%)$ \\
\hline Atenção especializada (ambulatorial/hospitalar) & $3(14,3 \%)$ & - & - & $3(7,6 \%)$ \\
\hline Vigilância & $1(4.7 \%)$ & - & - & $1(2,6 \%)$ \\
\hline $\begin{array}{l}\text { Educação Permanente/Professor/Preceptor/ } \\
\text { Coordenador de Ensino }\end{array}$ & $1(4.7 \%)$ & $4(44,4 \%)$ & $4(44.4 \%)$ & $9(23 \%)$ \\
\hline Organismo Internacional & $2(9.6 \%)$ & - & - & $2(5,2 \%)$ \\
\hline Planejamento/Articulação & $3(14.3 \%)$ & - & - & $3(7,6 \%)$ \\
\hline Consultor & $2(9.6 \%)$ & - & - & $2(5,2 \%)$ \\
\hline Secretário & $1(4.7 \%)$ & - & - & $1(2,6 \%)$ \\
\hline Médico de equipe saúde da família & - & $5(55.6 \%)$ & - & $5(12,8 \%)$ \\
\hline Pesquisador & - & - & $4(44.4 \%)$ & $4(10,2 \%)$ \\
\hline Estudante & - & - & $1(11.2 \%)$ & $1(2,6 \%)$ \\
\hline \multicolumn{5}{|l|}{ Nível de atuação } \\
\hline Municipal & $4(19 \%)$ & - & & \\
\hline Estadual & $5(23.8 \%)$ & - & & \\
\hline Federal & $6(28.6 \%)$ & - & & \\
\hline Serviço & $3(14.3 \%)$ & - & Não se aplica & Não se aplica \\
\hline Atenção básica & - & $8(88.9 \%)$ & & \\
\hline Atenção especializada (ambulatorial/hospitalar) & - & $1(11.1 \%)$ & & \\
\hline Não se aplica & $3(14.3 \%)$ & & & \\
\hline Tempo médio de trabalho na área (anos) & $11,3(7)$ & $11,5(7,7)$ & $16(13,21)$ & $12,5(9)$ \\
\hline \multicolumn{5}{|l|}{ Já participou de um Estudo Delphi } \\
\hline Sim & $6(28.6 \%)$ & $2(22.2 \%)$ & $5(55.5 \%)$ & $13(33.4 \%)$ \\
\hline Não & $15(71.4 \%)$ & $7(77.8 \%)$ & $4(44.5 \%)$ & $26(66.6 \%)$ \\
\hline
\end{tabular}

Fonte: Autoria Própria.

\section{Atenção Primária à Saúde}

A segunda dimensão, que abordava a APS, foi dividida em três subdimensões: aspectos gerais, aspectos relacionados à identificação das necessidades de saúde e integração com outros serviços da RAS e sistemas de informação da APS, totalizando 26 itens.

Destes 26, treze (50\%) (questões 9, 13, 16, 18, 19, 21, 22, 27, 28, 29, 30, 31, 32) tiveram, logo na segunda rodada, estabilidade e alto grau de consenso para inclusão no instrumento. Todas as questões relacionadas a sistemas de informação da APS estavam neste grupo, demonstrando o consenso em 


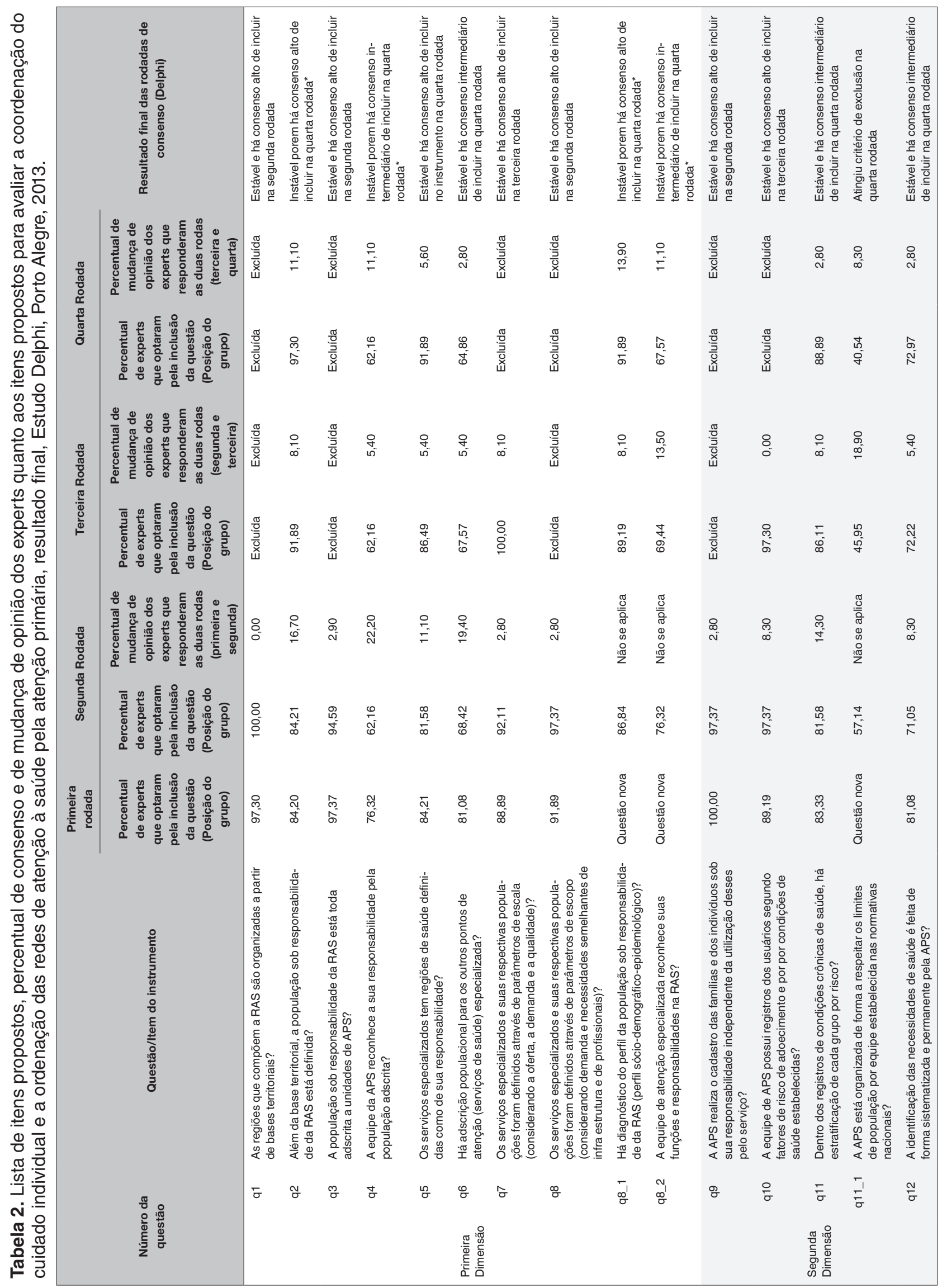




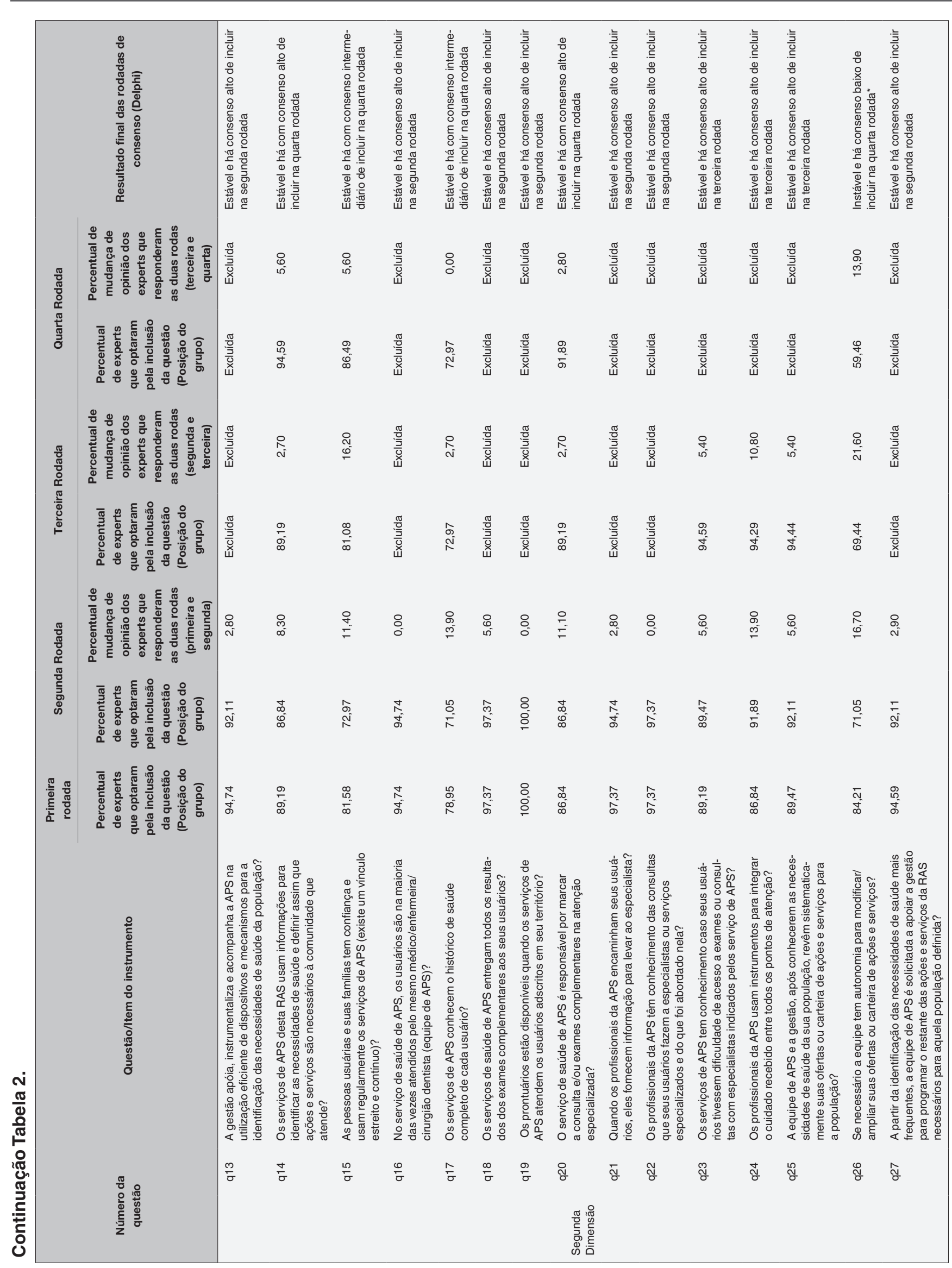




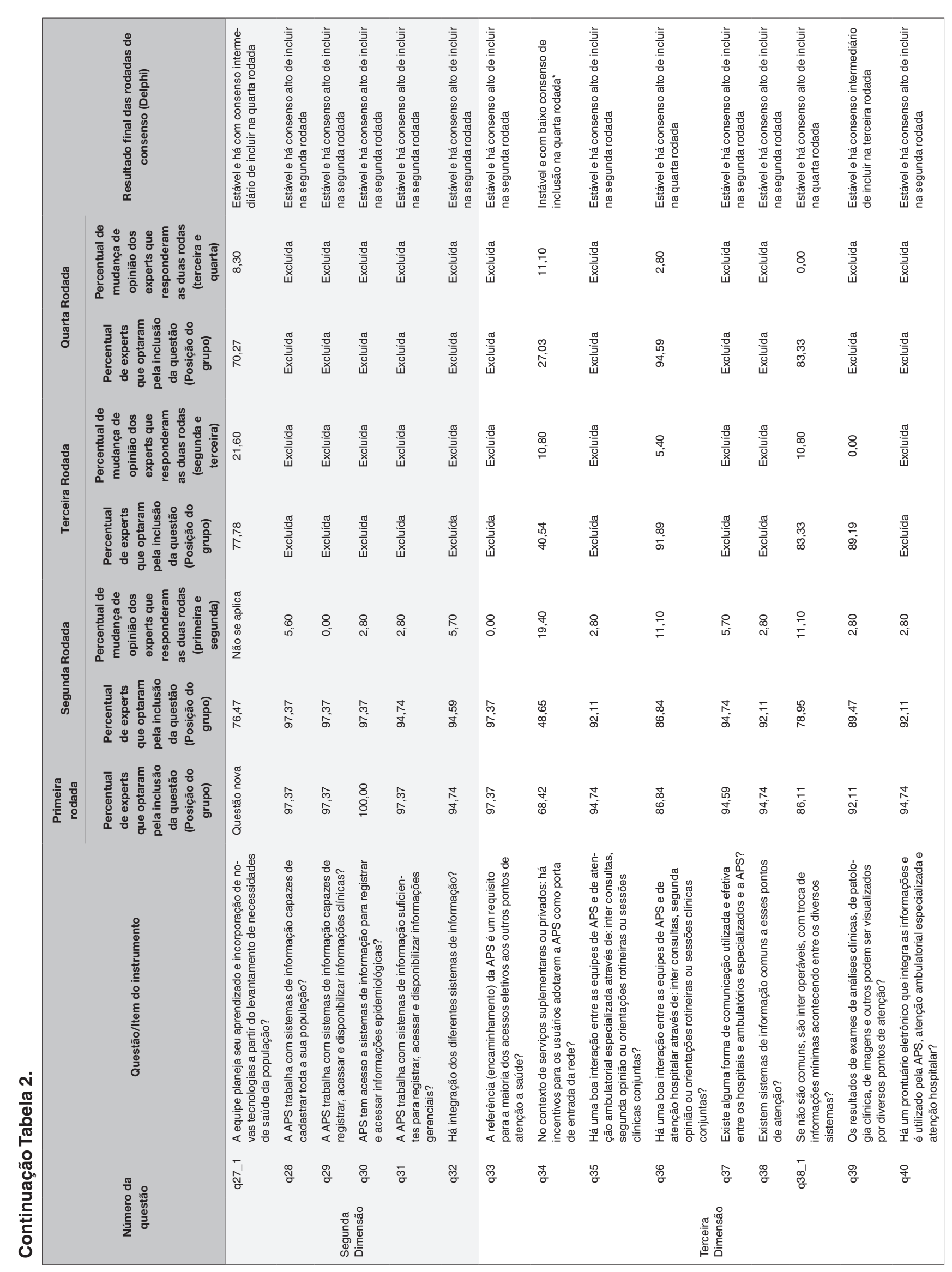




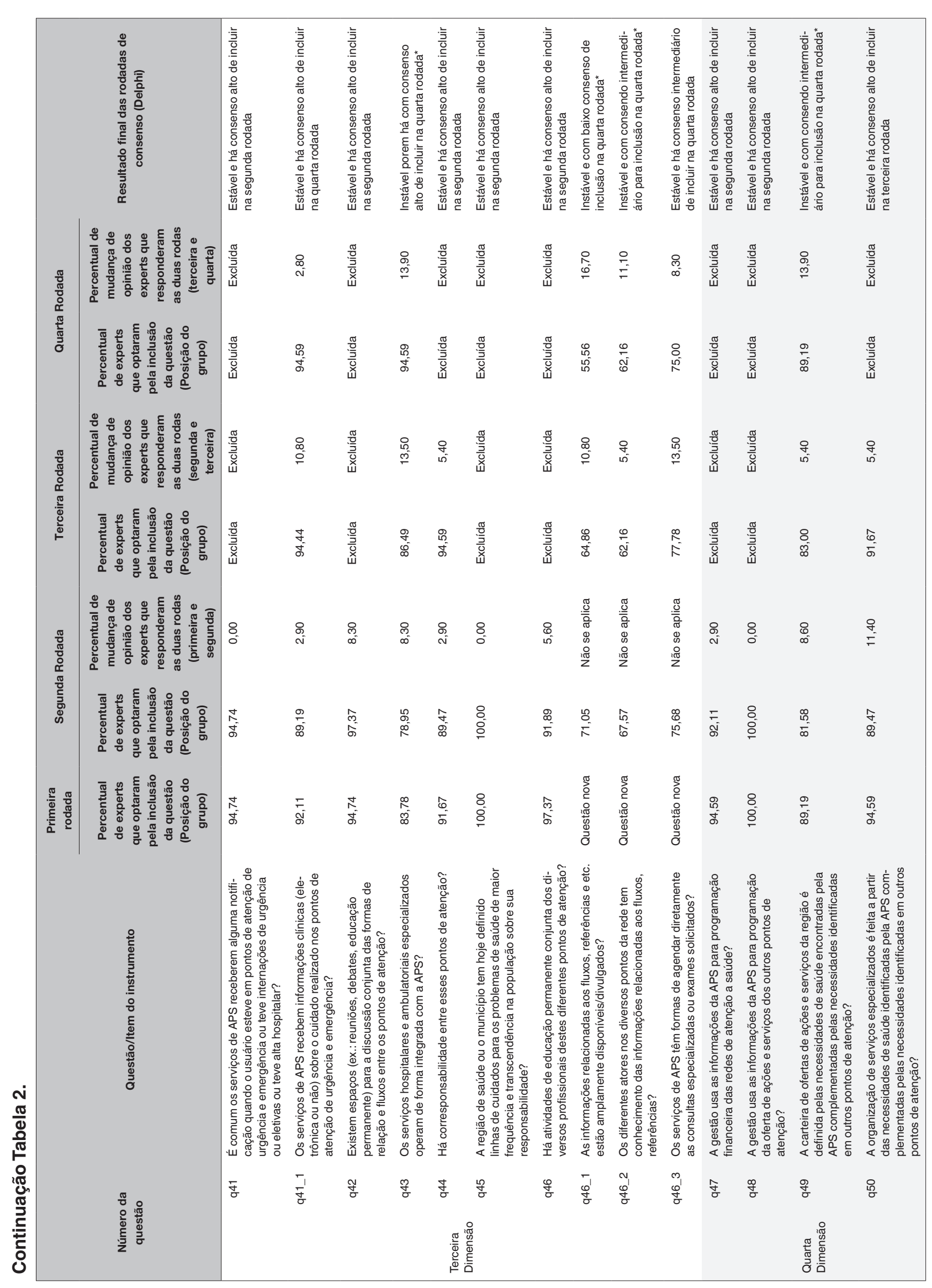




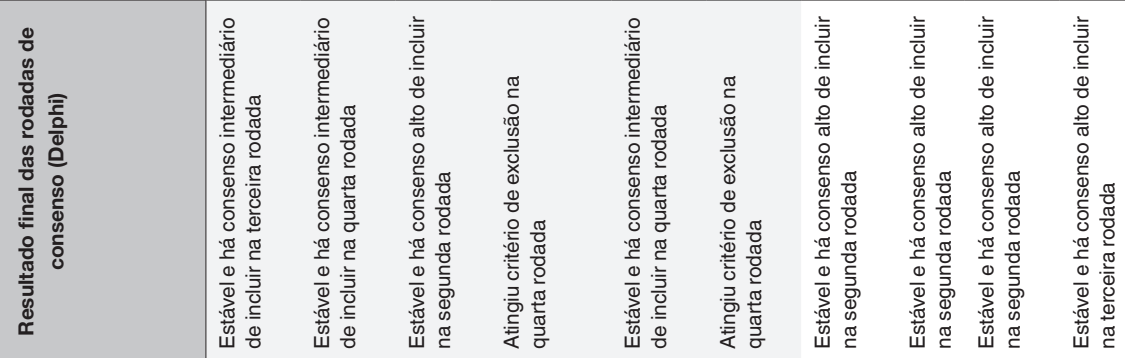

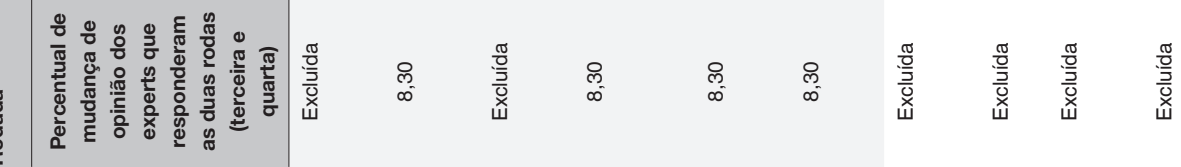

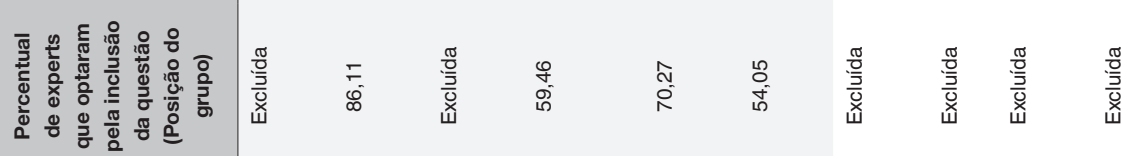

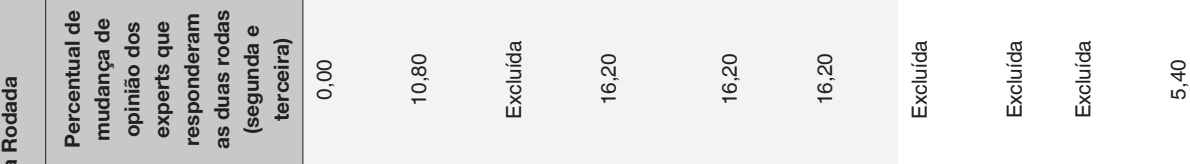

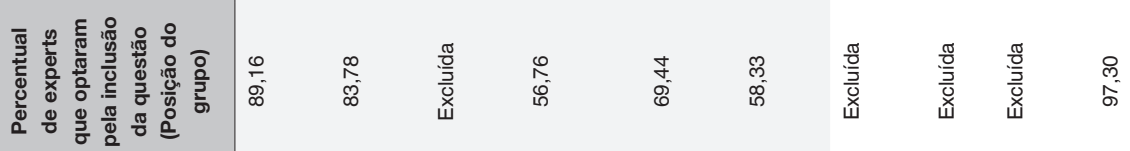

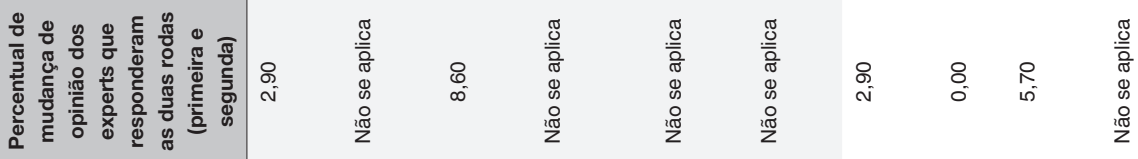

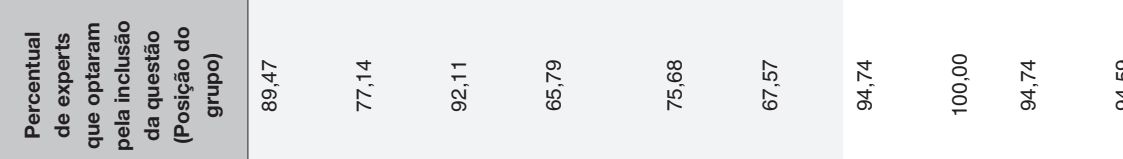

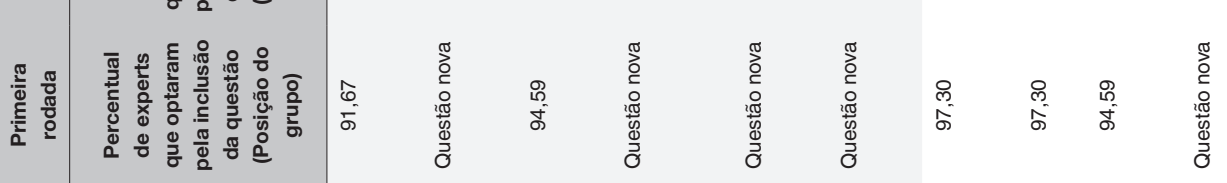

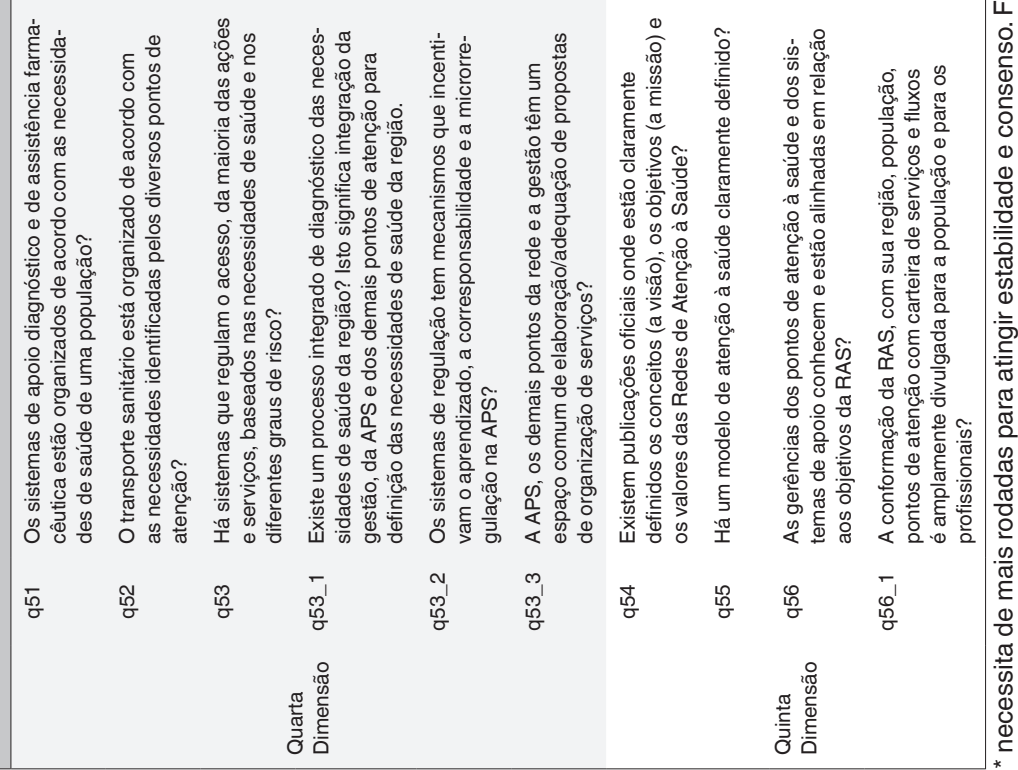


relação à essencialidade dos sistemas de informação para estruturação das RAS. Vários autores reforçam essa opinião, e apontam os sistemas de informação com importante papel para integração das ações e serviços da RAS. . $^{1-4,33}$

$\mathrm{Na} 3^{\mathrm{a}}$ rodada quatro questões $(10,23,24,25)$, e na $4^{\text {a }}$ rodada duas questões (14 e 20) atingiram estabilidade e o alto grau de consenso. Deste grupo, realçam-se as questões 14 e 25, que abordam a definição e a revisão sistemática da carteira de serviços da APS pela equipe em parceria com o gestor. Outras seis questões atingiram estabilidade na quarta rodada; porém, cinco delas (q11, q12, q15, q17, q27.1) tinham grau de consenso intermediário em relação à inclusão do item, e um único item (11.1), que versava sobre o limite populacional por equipe de APS, teve grau de consenso baixo para inclusão $(40,5 \%)$, atingindo critério de exclusão.

Ainda que a literatura não apresente critérios rígidos para limites populacionais por equipe/ médico, artigos colocam a orientação de que os serviços de APS tenham minimamente uma população definida. ${ }^{3,4,10,26,33} \mathrm{Na}$ visão dos pesquisadores o quantitativo populacional por equipe tem relação direta com a capacidade de resolução da equipe de APS e com o grau de coordenação do cuidado, portanto, apesar do baixo grau de consenso entre os experts, recomenda-se que este item componha o instrumento de avaliação das RAS.

Do grupo de consenso intermediário, chamam a atenção as questões 12, 15 e 17, que tratavam mais objetivamente de princípios da APS (vínculo e longitudinalidade), pois abordam temas não diretamente relacionados aos objetos do instrumento, mas considerados pressupostos para a consolidação da coordenação do cuidado e para a ordenação da RAS. É possível aventar a possibilidade de baixo consenso por não serem diretamente objeto de avaliação do instrumento.

Um único item (q26), que tratava da autonomia da equipe em rever a sua carteira de ações e serviços, não atingiu estabilidade em relação à opinião dos experts, e na última rodada do Delphi, tinha apenas $59,4 \%$ de opiniões a favor da sua inclusão e com uma variação de mudança de opinião na terceira e quarta rodada de 21,6\% e 13,9\%, respectivamente. Em 2006, Saltman et al. trataram da questão da autonomia profissional e da necessidade de regulação do processo de trabalho dos profissionais de saúde, ressaltando a importância de equilibrar essas duas ações para termos melhores resultados em saúde. ${ }^{5}$ Do mesmo modo, para os pesquisadores, o aspecto da autonomia profissional é essencial, pois pode, entre outras questões, aumentar a implicação da equipe com o trabalho. Portanto, os pesquisadores entendem que há necessidade de maior debate e pesquisa para definição de inclusão ou não deste item na avaliação das RAS.

\section{A articulação entre os pontos de atenção}

A terceira dimensão, que tratava da articulação entre os pontos de atenção, era composta de 19 itens. Destes, nove atingiram estabilidade e alto grau de consenso logo na segunda rodada (questões: $33,35,37,38,40,41,42,45,46$ ); exceto pelos itens 33 , que abordava a APS com porta de entrada, e o 45, que questionava sobre organização de linhas de cuidado, todos os demais tratavam de estratégias fundamentais para integração entre os diversos pontos de atenção (Tabela 2). 
O pronto consenso no grupo de experts, em relação aos itens que abordaram estratégias de integração, vai ao encontro de diversos autores, que incluem o aspecto integração como essencial para conformação das RAS e para avaliação de sistemas de saúde. 1-4,10,17,19-22,24-26,33

Quatro questões (q34, q43, q46.1 e.2) não atingiram estabilidade até a última rodada. As questões 46.2 e 46.1, que se referem à divulgação e ao conhecimento dos diversos fluxos na RAS pelos serviços, suscitaram a reflexão de que haveria necessidade de mais rodadas para que estas atingissem estabilidade; porém, decidiu-se parar o estudo Delphi na quarta rodada a fim de evitar a exaustão do grupo e uma possível diminuição na participação dos experts nas rodadas seguintes. ${ }^{29}$ Destas, ressalta-se a questão 34 ("No contexto de serviços suplementares ou privados: há incentivos para as pessoas usuárias adotarem a APS como porta de entrada da rede?"), que teve o grau de consenso mais baixo do estudo (27\%). Esse evento pode ter relação com o fato da amostra de experts ter apenas profissionais ligados ao sistema público.

Porém, diante da realidade do sistema de saúde brasileiro, que convive com os serviços privados, e da necessidade de qualificação deste setor, apesar da questão 34 não ter tido grau de consenso alto, os autores divergem do consenso dos experts e consideram que no futuro o item deva ser reavaliado para que o instrumento possa ser utilizado nos diferentes cenários de atenção à saúde do país. ${ }^{34}$

Programação da atenção à saúde a partir das necessidades em saúde

A quarta dimensão era composta de dez itens. Quatro questões atingiram estabilidade apenas na $4^{a}$ rodada; duas com grau de consenso intermediário, a 52, que tratava especificamente da organização do transporte na rede, e a 53.2, que abordava a regulação como apoio à gestão compartilhada e à educação permanente. Ambas trazem assuntos complexos de forma muito resumida, o que pode explicar o consenso entre os experts ter sido apenas intermediário. Há necessidade de revisão destas questões.

Além disso, duas questões tiveram baixo grau de consenso, a questão $53.1(59,4 \%)$ e a questão $53.3(54,6 \%)$, como tinham também instabilidade nas respostas, preencheram critérios para exclusão. Ambas versavam sobre a integração dos serviços visando o diagnóstico das necessidades de saúde e o planejamento, e têm um caráter mais operacional. São apontadas como característica fundamental para os serviços de saúde se conformarem em rede por diversos autores ${ }^{1,4,33}$ e consideradas essenciais pelos pesquisadores.

Compromisso da gestão com a organização das redes

A quinta e última dimensão, que tratava dos conceitos, da divulgação e do compromisso da gestão com a organização da RAS, era composta de quatro questões. Esta foi a única dimensão em que todos os itens tiveram estabilidade e alto grau de consenso.

Proposta de itens a comporem um instrumento de avaliação das RAS

Ao final das quatro rodadas, resultou um instrumento com 69 itens. Do total de questões, apenas dez $(14,5 \%)$ não atingiram grau de estabilidade até a última rodada. Os detalhes sobre o comportamento de cada item podem ser observados na Tabela 2. 


\section{Considerações finais}

A construção do instrumento apresentou algumas limitações, parte destas ligada ao tema "redes de atenção à saúde", que ainda não possui um descritor, dificultando a revisão da literatura. Além disso, as diversas nomenclaturas relacionadas ao tema foram um obstáculo para a avaliação integral do assunto. 2,4,24,35

A revisão de literatura realizada não se caracterizou por ser sistemática, e, portanto, não teve a pretensão de esgotar o assunto. Acredita-se, porém, que a diversificada composição do grupo de experts, com representantes das áreas de gestão, assistência e academia, de quase todas as regiões do país (Sul, Sudeste, Nordeste e Centro-Oeste), atenue esta limitação.

A metodologia Delphi não prevê a interação dos participantes, visando impedir "contaminação" das opiniões, mas alguns participantes expressaram que a interação poderia ter contribuído com as discussões e com o alcance de maior consenso.

O resultado final não difere extremamente da versão inicial proposta pelos pesquisadores: algumas questões foram apuradas e outras incluídas com pouca agregação de novos conteúdos. Assim, é possível dizer que nessa pesquisa o método Delphi teve a função de legitimar, perante o meio acadêmico, a proposta inicial feita pelos pesquisadores, que foi consensuada e aperfeiçoada por experts no assunto.

O resultado final não deve ser considerado um instrumento pronto para ser aplicado, há necessidade de dar sequência ao projeto, aprimorando-o e associando outras metodologias e testes de validação, e, assim, ofertar um instrumento validado que permita a comparação das características e estágios de organização de redes de atenção à saúde. Para que no futuro seja possível avaliar o impacto da organização das RAS nos indicadores de saúde da população.

\section{Referências}

1. Hartz ZMA, Contandriopoulos AP. Integralidade da atenção e integração de serviços de saúde: desafios para avaliar a implantação de um "sistema sem muros". Cad Saúde Publica. 2004;20(Suppl. 2):S331-6.

2. Kodner DL. All together now: a conceptual exploration of integrated care. Healthc Q. 2009;Spec No:6-15.

3. Mendes EV. As redes de atenção à saúde. 2ª ed. Brasília: Organização Pan-Amaericana da Saúde; 2011.549 p.

4. Organización Panamericana de la Salud - OPAS. Redes Integradas de Servicios de Salud. Conceptos, opciones de política y hoja de ruta para su implementación en las Américas [Internet]. Serie Renovacion de la Atencion Primaria de Salus en las Américas - Organizacion Panamericana de la Salud No.4. Washington: OPAS; 2010. [cited 2013 Jan 12]. p. 102. Available from: http://scholar.google.com/schola $r ? h l=e n \& b t n G=S e a r c h \& q=i n t i t l e: R e d e s+I n t e g r a d a s+d e+S e r v i c i o s+d e+S a l u d:+C o n c e p r o s,+o p c i o n e s+d e+p o l i t i c a+y+h o j a+d e+r u t a+p$ ara+su+implementacíon+en+las+Américas\#0

5. Saltman RB, Rico A, Boerma WGW, eds. Primary care in the driver's seat? Organizational reform in European primary care. Maidenhead: Open Universitary Press; 2006.

6. Brasil. Minstério da Saúde. Conselho Nacional de Saúde B. O desenvolvimento do Sistema Único de Saúde: avanços, desafios e reafirmação dos seus princípios e diretrizes [Internet]. Brasília: Ministério da Saúde; 2003. [citado 2013 Mar 29]. Disponível em: http:// livroaberto.ibict.br/handle/1/890

7. Brasil. Ministério da Saúde. Norma Operacional Básica do Sistema Único de Saúde/NOB SUS 96. Brasília: Ministério da Saúde; 1997.

8. Brasil. Ministério da Saúde. Portaria n 4279 de dezembro de 2010. Estabelece Diretrizes para a Organização da Rede de Atenção à Saúde no Ambito do Sistema Único de Saúde (SUS). Brasília: Ministério da Saúde; 2010. 
9. Brasil. Ministério da Saúde. Regionalização da Assistencia à Saúde: Aprofundando a Descentralização com Equidade no Acesso: Norma Operacional da Assisência à Saúde: NOAS-SUS 01/02 (Portaria MS/GM n. 373, de 27 de fevereiro de 2002 e regulamentação complementar). Brasília: Minstério da Saúde; 2002.

10. Carvalho G, Magalhães Júnior HM, Medeiros L, Souto Júnior JV, Santos L, Andrade LOM, et al. Redes de atenção à saúde no SUS: o pacto pela saúde e redes regionalizadas de ações e serviços de saúde [Internet]. Campinas: IDISA CONASEMS; 2008.202 p. Available from: http://relativa.com.br/livros_template.asp?Codigo_Produto=154708

11. Brasil. Constituição da República Federativa do Brasil [Internet]. Brasília: Senado Federal; 1988 [cited 2013 Mar 29]. Available from: https://ensinolegal.s3.amazonaws.com/uploads/legislation/file/29/Constitui_o_da_Rep_blica_Federativa_do_Brasil.pdf

12. Brasil. Decreto Presidencial no. 7508 de junho de 2011. Regulamenta a Lei no 8.080, de 19 de setembro de 1990, para dispor sobre a organização do Sistema Único de Saúde - SUS, o planejamento da saúde, a assistência à saúde e a articulação interfederativa, e dá outras providências. Brasília: Presidência da República; 2011.

13. Noronha JO, Lima LD, Machado CV. O Sistema Único de Saúde: SUS. In: Giovanella L, Escorel S, Lobato, LVC, Noronha JC, Carvalho Al, orgs. Políticas e sistema de saúde no Brasil. 2a ed. Rio de Janeiro: Fiocruz; 2012. p.1100.

14. Assis MMA, Cerqueira EM, Nascimento MAA, Santos AM, Abreu de Jesus WL. Atenção Primária à Saúde e sua articulação com a Estratégia Saúde da Família: construção política, metodológica e prática. Rev APS. 2007;10(2):189-99.

15. Campos GWS, Guerrero AVP, orgs. Manual de Prática de atenção básica e a estratégia saúde da família. 411 p. [cited 2017 Jul 12 ]. Available from: http://andromeda.ensp.fiocruz.br/teias/sites/default/files/biblioteca_home/manual_das_praticas_de_atencao_basica\%5B1\%5D. pdf

16. Brasil. Ministério da Saúde. Lei 12.871 de 22 de outubro de 2013. Institui o Programa Mais Médicos, altera as Leis no 8.745 , de 9 de dezembro de 1993, e no 6.932, de 7 de julho de 1981, e dá outras providências. Brasília: Diário Oficial da União; 2013.

17. Harzheim E. OPAS - Organização Pan-Americana da Saúde. Inovando o papel da atenção primária nas redes de atenção à saúde: resultados do laboratório de inovação em quatro capitais brasileiras. $1^{\text {a }}$ ed. Organização Pan-Americana da Saúde, Ministério da Saúde. Brasília: OPAS; 2011.

18. Campos GWS, Júnior NP. A Atenção Primária e o Programa Mais Médicos do Sistema Único de Saúde: conquistas e limite. Ciênc Saúde Coletiva. 2016;21(9):2655-63.

19. Starfield B. Atenção Primária: equilíbrio entre necessidades de saúde, serviços e tecnologias. Brasília/UNESCO: Ministério da Saúde; 2002.

20. Davies GP, Harris M, Perkins D, Roland M, Williams A, Larsen K, et al. Coordination of care within primary health care and with other sectors: a systematic review. Camberra: Australian Primary Health Care Research Institute; UNSW Research Centre For Primary Health Care And Equity (CPHCE) at the University of New South Wales in Association With the University of Manchester (UK); 2006.141 p.

21. Bodenheimer T. Coordinating care--a perilous journey through the health care system. N Engl J Med. 2008;358(10):1064-71.

22. Hofmarcher MM, Oxley H, Rusticelli E. Improved health system performance throuch better care coordenation. Paris: OECD; 2007.

23. Schoen C, Osborn R, Squires D, Doty M, Pierson R, Applebaum S. New 2011 survey of patients with complex care needs in eleven countries finds that care is often poorly coordinated. Health Aff (Millwood). 2011;30(12):2437-48.

24. Armitage GD, Suter E, Oelke ND, Adair CE. Health systems integration: state of the evidence. Int J Integr Care. 2009;9:e82.

25. Waddington C, Egger D. Integrated Health Services—What and why? Geneva: World Health Organization; 2008.

26. Shortell SM, Gillies RR, Anderson DA. The new world of managed care: creating organized delivery systems. Health Aff (Millwood). 1994;13(5):46-64.

27. Organização Pan-Americana da Saúde - OPAS. A atenção à saúde coordenada pela APS: construindo as redes de atenção no SUS: contribuições para o debate. Brasília: Organização Pan-Americana da Saúde; 2011.

28. Starfield B. Primary care: an increasingly important contributor to effectiveness, equity, and efficiency of health services. SESPAS report 2012. Gac Sanit. 2012;Suppl1:20-6.

29. Brasil. Ministério da Saúde. Política Nacional de Atenção Básica. Secretaria de Atenção à Saúde. Departamento de Atenção Básica. Brasília: Ministério da Saúde; 2012. 
30. McDonald KM, Schultz E, Albin L, Pineda N, Lonhart J, Sundaram V, et al. Care Coordination Measures Atlas. Version 3. Rockville: Agency for Healthcare Research and Quality; 2010.

31. Keeney S, McKenna H, Hasson F. The Delphi Technique in Nursing and Health Research. $1^{\text {st }}$ ed. Chichester: Wiley-Blackwell; 2011.

32. Fontanive PVN. Necessidades em educação permanente percebidas por profissionais médicos das equipes da estratégia saúde da família dos municípios do projeto Telessaúde-RS [Dissertação de mestrado]. Porto Alegre: Universidade Federal do Rio Grande do Sul; 2009.

33. Suter E, Oelke ND, Adair CE, Armitage GD. Ten key principles for successful health systems integration. Healthc Q.2009;13:16-23.

34. Brasil. Agencia Naiconal de Saúde Suplementar. Qualificação da Saúde Suplementar: Uma nova perspectiva no processo de regulação. Rio de Janeiro: Agencia Nacional de Saúde Suplementar; 2010.

35. Conill EM, Fausto MCR, Giovanella L. Contribuições da análise comparada para um marco abrangente na avaliação de sistemas orientados pela atenção primária na América Latina. Rev Bras Saúde Mater Infant. 2010;10(Suppl.1):S14-27.

a Departamento de medicina social da Faculdade de Medicina da Universidade Federal do Rio Grande do Sul (UFRGS); Programa de Pós Graduação em Epidemiologia da Universidade Federal do Rio Grande do Sul (UFRGS). Porto Alegre, RS, Brasil. patriciachueiri@gmail.com (Autora correspondente); erno.harzheim @ telessauders.ufrgs.br

• Serviço de Saúde Comunitária do Hospital Nossa Senhora do Grupo Hospitalar Conceição. Porto Alegre, RS, Brasil. tsilvia@ghc.com.br 